QCD Evolution Workshop 2013

International Journal of Modern Physics: Conference Series

Vol. 25 (2014) 1460033 (9 pages)

(C) The Author

DOI: $10.1142 / \mathrm{S} 2010194514600337$

\title{
RECENT RESULTS ON HIGH-ENERGY SPIN PHENOMENA OF GLUONS AND SEA-QUARKS IN POLARIZED PROTON-PROTON COLLISIONS AT RHIC AT BNL*
}

\author{
BERND SURROW \\ Temple University, Department of Physics, Philadelphia, PA, USA \\ surrow@temple.edu
}

Published 2 January 2014

\begin{abstract}
The STAR experiment at the Relativistic Heavy-Ion Collider at Brookhaven National Laboratory is carrying out a spin physics program in high-energy polarized proton collisions at $\sqrt{s}=200 \mathrm{GeV}$ and $\sqrt{s}=500 \mathrm{GeV}$ to gain a deeper insight into the spin structure and dynamics of the proton.

One of the main objectives of the spin physics program at RHIC is the precise determination of the polarized gluon distribution function. The STAR detector is well suited for the reconstruction of various final states involving jets, $\pi^{0}, \pi^{ \pm}, \mathrm{e}^{ \pm}$and $\gamma$, which allows to measure several different processes. Recent results suggest a gluon spin contribution to the proton spin at the same level as the quark spin contribution itself.

The production of $W$ bosons in polarized $\mathrm{p}+\mathrm{p}$ collisions at $\sqrt{s}=500 \mathrm{GeV}$ opens a new era in the study of the spin-flavor structure of the proton. $W^{-(+)}$bosons are produced in $\bar{u}+d(\bar{d}+u)$ collisions and can be detected through their leptonic decays, $e^{-}+\bar{\nu}_{e}\left(e^{+}+\nu_{e}\right)$, where only the respective charged lepton is measured. Results of $W^{-(+)}$production suggest a large asymmetry between the polarization of anti- $u$ and anti- $d$ quarks.
\end{abstract}

Keywords: BNL; RHIC; STAR; pp collisions; QCD; proton spin; $A_{L}$; quark polarization; antiquark polarization; global analysis.

PACS numbers: 12.38.-t, 13.85.Ni, 13.87.-a, 13.87.Fh, 13.88.+e, 14.70.Dj

\section{Introduction}

High energy polarized $p+p$ collisions at a center-of-mass energy of $\sqrt{s}=200 \mathrm{GeV}$ and at $\sqrt{s}=500 \mathrm{GeV}$ at RHIC at BNL provide a unique way to probe the proton spin structure using very well established processes in high-energy physics, both experimentally and theoretically. The polarized gluon and quark / antiquark measurements using jet and $\mathrm{W}$ boson production at RHIC have been formulated

*This is an Open Access article published by World Scientific Publishing Company. It is distributed under the terms of the Creative Commons Attribution 3.0 (CC-BY) License. Further distribution of this work is permitted, provided the original work is properly cited. 
as key milestones of the US Nuclear Physics program. A recent global analysis provides for the first time evidence of a non-zero value of the gluon polarization $\int_{0.05}^{0.2} \Delta g d x\left(Q^{2}=10 \mathrm{GeV}^{2}\right)=0.1_{-0.07}^{+0.06}$, i.e. of similar magnitude as the total quark spin contribution to the proton spin. The analysis of data in 2009 using for the first time collisions of polarized protons at $\sqrt{s}=500 \mathrm{GeV}$ established a novel scheme to probe the quark flavor structure using $W$ boson production. Data taken in 2012 suggest a large asymmetry of the spin contribution of anti-u quarks and anti-d quarks similar to the large and well-known difference between the momentum distributions of anti-u quarks and anti-d quarks. Highlights of these results will be presented in the following two sections.

\section{High-Energy Spin Phenomena of Gluons}

The gluon polarization program is based on the measurement of various probes, such as inclusive jet production and di-jet production in polarized proton-proton collisions at RHIC using the STAR experiment. The full exploitation of the STAR Barrel and Endcap Electromagnetic Calorimeter (BEMC / EEMC) is crucial for this analysis effort. Both the first measurement at STAR of the longitudinal double-spin asymmetry for inclusive jet production ${ }^{1}$ and updates with higher precision ${ }^{2,3}$ along with the first neutral pion measurement ${ }^{4}$ have been published. Precision measurements of inclusive jet production ${ }^{5}$ along with the first significant di-jet measurements ${ }^{6}$ have been presented suggesting a statistically non-zero gluon spin contribution to the proton spin at the level of $\int_{0.05}^{0.2} \Delta g d x\left(Q^{2}=10 \mathrm{GeV}^{2}\right)=0.1_{-0.07}^{+0.06}$, i.e. of similar magnitude as the total quark spin contribution to the proton spin.

The measurement of the gluon polarization through inclusive measurements such as jet production and $\pi^{0}$ and $\pi^{ \pm}$production was the prime focus of the physics analysis program of the Run $3 / 4$ and Run 5 data samples as well as the Run 6 and Run 9 data samples. The sensitivity of those inclusive measurements to the underlying gluon polarization in high-energy polarized proton-proton collisions has been discussed in detail in Refs. [7, 8, 9].

The 2006 inclusive jet cross-section measurement is shown in Figure 1 (a) [10]. Good agreement is found between data and full NLO calculations taking into account corrections for underlying event and hadronization effects. Figure 1 (b) shows the most recent STAR preliminary result of $A_{L L}$ for inclusive jet production as a function of $p_{T}$ based on the 2006 and 2009 data sample. The 2006 analysis has been published. ${ }^{3}$ The 2009 data fall in-between the GRSV-STD $\left(\Delta G=\int_{0}^{1} \Delta g d x\left(Q^{2}=10 \mathrm{GeV}^{2}\right)=0.4\right)[11]$ and DSSV $\left(\Delta G=\int_{0}^{1} \Delta g d x\left(Q^{2}=\right.\right.$ $\left.10 \mathrm{GeV}^{2}\right)=0.0$ ) [12] NLO calculations for $A_{L L}$. The DSSV fit result was constrained by the 2005 [2] and 2006 [3] data. The 2009 result suggests a larger gluon polarization than the one suggested by DSSV, driven by the low- $p_{T}$ region. Prior to a full global analysis incorporating the 2009 result, a first step to quantify the impact of the 2009 result has been made by W. Vogelsang et al. [13]. This study suggests a non-zero gluon polarization at the level of $\int_{0.05}^{0.2} \Delta g d x\left(Q^{2}=10 \mathrm{GeV}^{2}\right)=0.13$ and 
(a)

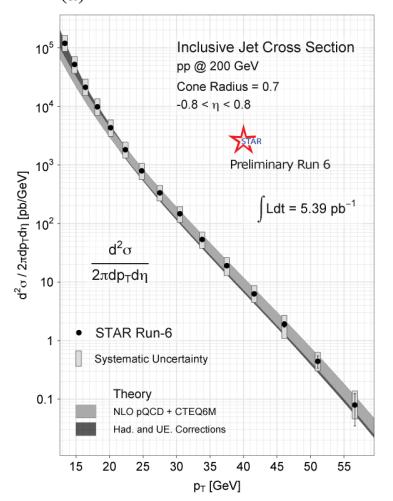

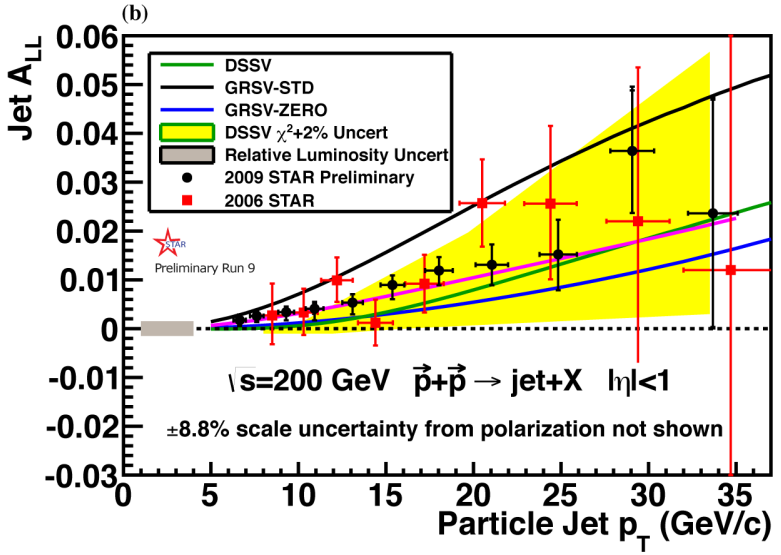

Fig. 1. Cross-section based on STAR Run 6 data (a) and longitudinal double-spin asymmetry based on STAR Run 9 data (b) for inclusive jet production as a function of $p_{T}$ in comparison to NLO calculations.

thus of similar magnitude than the quark spin contribution, $\frac{1}{2} \Delta \Sigma$, to the proton spin.

Di-jet production at STAR will allow a better constraint of the underlying event kinematics to extract the shape of the gluon polarization in a global analysis. ${ }^{14}$ The invariant di-jet mass is proportional to the sum of the partonic Bjorken-x values, $\mathrm{x}_{1}+\mathrm{x}_{2}$, whereas the pseudo-rapidity sum $\eta_{3}+\eta_{4}$ is proportional to the logarithmic ratio of the partonic Bjorken-x values, $\log \left(\mathrm{x}_{1} / \mathrm{x}_{2}\right)$. Measurements at both $\sqrt{\mathrm{s}}=200 \mathrm{GeV}$ and $\sqrt{\mathrm{s}}=500 \mathrm{GeV}$ are preferred to maximize the kinematic region in $\mathrm{x}$. The wide acceptance of the STAR experiment permits reconstruction of di-jet events with different topological configurations, i.e. different $\eta_{3} / \eta_{4}$ combinations, ranging from symmetric $\left(x_{1}=x_{2}\right)$ partonic collisions to asymmetric $\left(x_{1}<x_{2}\right.$ or $x_{1}>x_{2}$ ) partonic collisions (Figure 2 (e) and (f)). This, together with the variation of the center-of-mass energy, is expected to constrain $\Delta g$ over a wide range in $x$ of approximately $\simeq 3 \cdot 10^{-3}<x<0.3$. The NLO framework for correlation measurements exists and therefore those measurements can be used in a global analysis. ${ }^{15}$ The needed extension from the first di-jet measurement in 2009 at mid-rapidity to the forward rapidity region requires an extension of jet reconstruction into the STAR EEMC acceptance region $(1<|\eta|<2)$. The utilization of the STAR Forward GEM Tracker to provide tracking information in addition to the calorimetric information provided by the STAR EEMC will be important. Figure 2 (a) shows the first di-jet cross-section measurement. ${ }^{16}$ Good agreement is found between data and full NLO calculations taking into account corrections for underlying event and hadronization effects. Figures 2 (b) - (d) show the first significant measurement of the longitudinal double-spin asymmetry, $A_{L L}$, as a function of the di-jet invariant mass, $M$, for different pseudo-rapidity regions (2 (e) and (f)) emphasizing different Bjorken- $x$ regions. The 2009 di-jet $A_{L L}$ measurement also suggests a larger gluon 
(a)

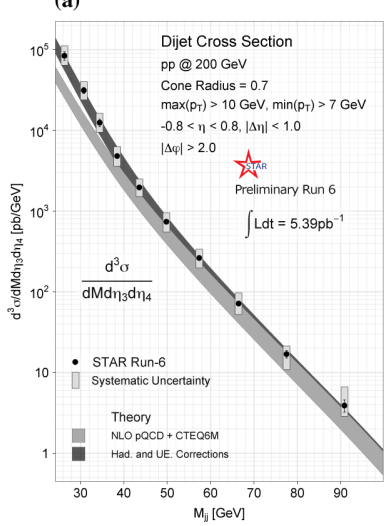

(b)
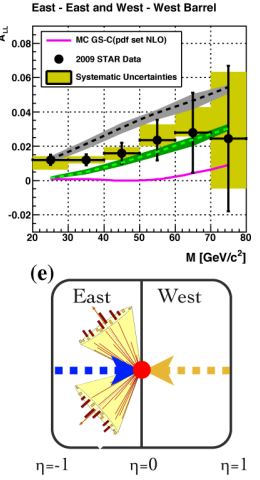

(c)
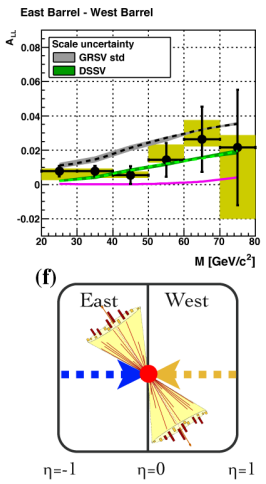

(d)

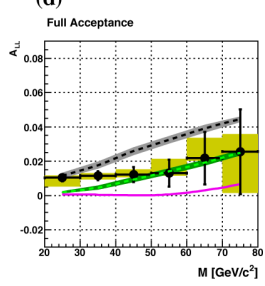

Fig. 2. Cross-section (a) and longitudinal double-spin asymmetry for three different $\eta$ regions (b), (c) and (d) for di-jet production as a function of $M$ based on Run 9 STAR data in comparison to NLO calculations. Illustration of asymmetric (e) and symmetric $(f)$ collisions.

polarization scenario than those suggested by DSSV [12]. The data fall in-between the GRSV-STD [11] and DSSV [12] polarized PDFs used for a full NLO calculation similar to the inclusive-jet result shown in Figure 1.

All current PHENIX and STAR $A_{L L}$ measurements taken together, in comparison to different NLO perturbative QCD predictions for $A_{L L}$, provide a consistent picture which rules out a large gluon polarization scenario. At the same time, data taken in Run 9 provide the first evidence for a non-zero gluon spin contribution to the proton spin. A critical aspect to extract the gluon polarization of the proton is to perform a global analysis of several $A_{L L}$ measurements obtained from the PHENIX and STAR collaborations, taking into account a constraint of polarized PDFs at high Bjorken-x values by several polarized fixed-target DIS experiments.

Figure 4 shows the impact of the Run 9 and future data sets on the polarized gluon distribution function. ${ }^{17}$ The Run 9 result suggests a statistically non-zero value of the gluon polarization at the level of $\int_{0.05}^{0.2} \Delta g d x\left(Q^{2}=10 \mathrm{GeV}^{2}\right)=0.1_{-0.07}^{+0.06}$ which is of similar magnitude as the total quark spin contribution itself. Future

(a)

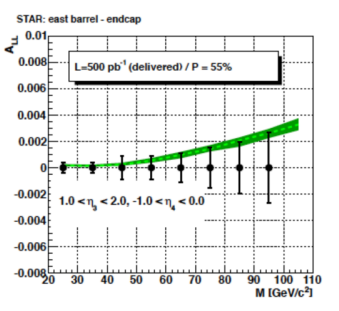

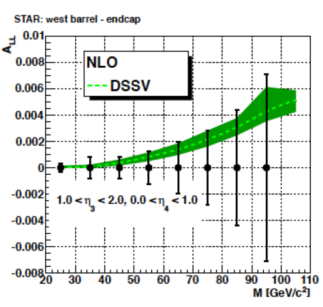

(b)

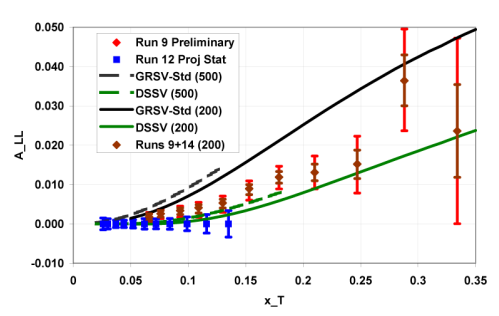

Fig. 3. Projection of STAR longitudinal double-spin asymmetry measurements for di-jet (a) and inclusive jet (b) production. 
(a)

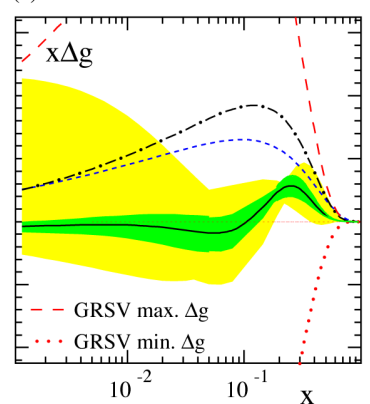

(b)

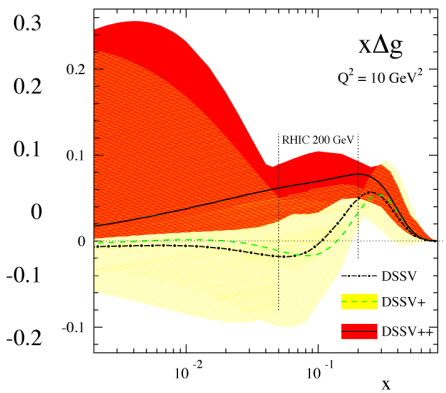

(c)

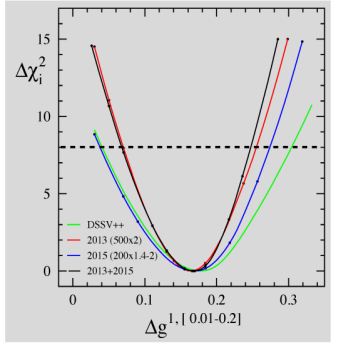

Fig. 4. Impact of the Run 9 STAR inclusive jet and PHENIX neutral pion results on the polarized gluon distribution function (a) and (b) together with the $\Delta \chi^{2}$ distribution as a function of the truncated first moment of $\Delta g$ restricted to $0.05<x<0.2$. The achieved precision is at least improved by a factor 2 compared to the original DSSV uncertainty band. ${ }^{12}$

(a)

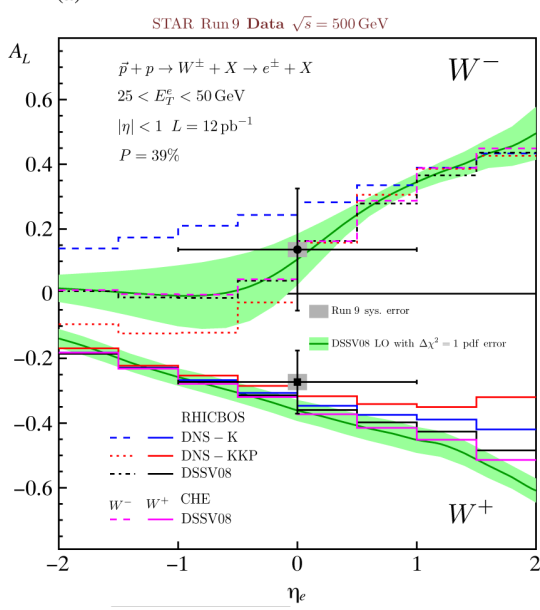

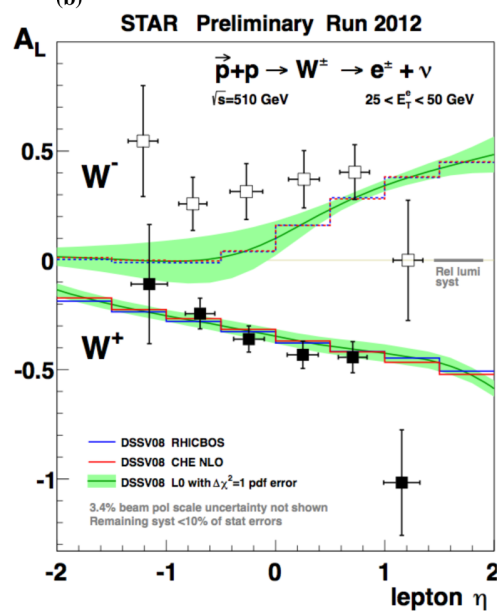

Fig. 5. First STAR longitudinal single-spin asymmetry for $W^{ \pm}$production based on Run 9 data (a) and preliminary results from Run 12 (b).

measurements will focus on extending the range towards smaller values in Bjorken$x$ and providing higher precision measurements at the level of a factor of 2 compared to the original DSSV fit uncertainties. ${ }^{12}$

\section{High-Energy Spin Phenomena of Sea-Quarks}

The data taking period in 2009 of polarized $p+p$ collisions at $\sqrt{s}=500 \mathrm{GeV}$ opened a new era in the study of the spin-flavor structure of the proton based on the production of $W^{-(+)}$bosons. $W^{-(+)}$bosons are produced predominantly through $\bar{u}+d$ $(u+\bar{d})$ collisions and can be detected through their leptonic decay. ${ }^{18}$ Quark and antiquark polarized parton-distribution functions (PDFs) are probed in calculable 


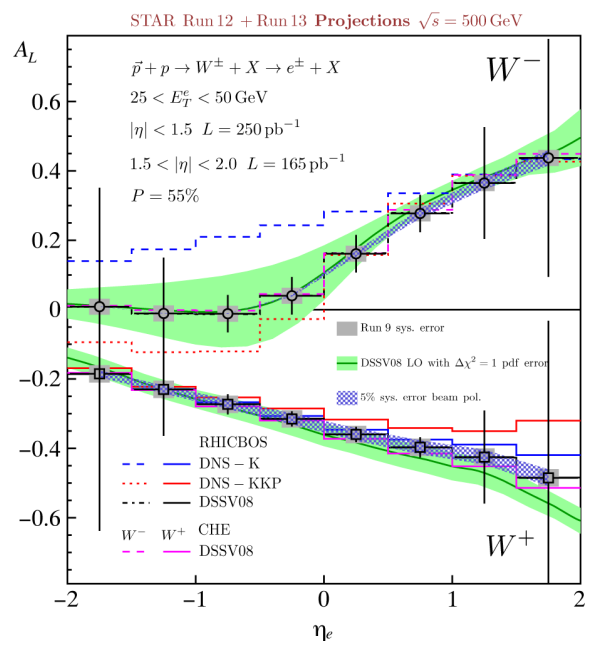

Fig. 6. Projected longitudinal single-spin asymmetry for $W$ production as a function of $\eta$.

leptonic $W$ decays at large scales set by the mass of the $W$ boson. The production of $W$ bosons in polarized proton collisions allows for the observation of purely weak interactions, giving rise to large, parity-violating, longitudinal single-spin asymmetries. A theoretical framework has been developed to describe inclusive lepton $(l)$ production, $p+p \rightarrow W^{ \pm}+X \rightarrow l^{ \pm}+X$, that can be directly compared with experimental measurements using constraints on the transverse energy and pseudorapidity of the final-state leptons. ${ }^{19,20}$ This development profits from a rich history of hadroproduction of weak bosons at the CERN SPS and the FNAL Tevatron and provides a firm basis to use $W$ production as a new high-energy probe in polarized $p+p$ collisions. ${ }^{21}$ The subsequent analyses of both the $\mathrm{W}$ boson asymmetry and cross-section measurements have been published. ${ }^{22,23}$ The first cross-section measurement is shown in Figure 7. Data taken in 2012 suggest a large asymmetry of the spin contribution of anti-u quarks and anti-d quarks similar to the large and well-known asymmetry between the momentum distributions of anti-u quarks and anti-d quarks. ${ }^{24}$

An upgrade of the STAR forward tracking system was carried out with the installation of the Forward GEM Tracker (FGT) in 2012 prior to the 2013 data taking period. ${ }^{25}$ This upgrade will improve the ability of the STAR tracking system to track high-energetic electrons (positrons) from $W^{-(+)}$-boson decays at forward acceptance enhancing the sensitivity to quark / antiquark polarizations.

The first published $W$ measurement at mid-rapidity using a Run 9 data sample is based on $L=12 \mathrm{pb}^{-1}$ [22]. This achievements marks a major milestone of the RHIC Spin program, in particular the ability of the STAR detector system to establish the reconstruction of $W$ bosons, both for a cross-section and asymmetry measurement. The STAR detector systems used in this analysis are the STAR Time Projection Chamber (TPC), the STAR Barrel Electromagnetic Calorimeter (BEMC) and the 
STAR Electromagnetic Endcap Calorimeter (EEMC). Only their tower response has been taken into account. The BEMC was used to measure the transverse energy, $E_{T}$, of $e^{ \pm}$. The suppression of QCD background by several orders of magnitude was based on the TPC, BEMC and EEMC. The STAR TPC plays a critical role to provide an efficient way for high- $p_{T}$ charge-sign discrimination of electron / positron track candidates. The reconstruction of $W$ boson events is well established including the treatment of background. This will be the basis for future measurements, in particular the large Run 13 data sample of $L \simeq 300 \mathrm{pb}^{-1}$. The critical new step is the development of tracking software concerning the charge-sign separation of high- $p_{T}$ electrons (positrons) from $W^{-(+)}$-decays for $1<|\eta|<2$ using the STAR FGT.

Figure 5 (a) shows the measured leptonic asymmetries $A_{L}^{W^{+}}$and $A_{L}^{W^{-}}$for $\left|\eta_{e}\right|<1$ and $25<E_{T}^{e}<50 \mathrm{GeV}$ from Run 9. The vertical black error bars include only the statistical uncertainties. The systematic uncertainties are indicated as grey bands. The statistical uncertainties dominate over the systematic uncertainties. The measured asymmetries are compared to predictions based on full resummed (RHICBOs) [19] and NLO (CHE) [20] calculations. The CHE calculations use the DSSV08 polarized PDFs [12], whereas the RHICBOs calculations are shown in addition for the older DNS-K and DNS-KKP [26] PDFs. The CHE and RHICBOs results are in good agreement. The range spanned by the DNS-K and DNS-KKP distributions for $\Delta \bar{d}$ and $\Delta \bar{u}$ coincides, approximately, with the corresponding DSSV08 uncertainty estimates. ${ }^{12,27}$ The spread of predictions for $A_{L}^{W^{+(-)}}$is largest at forward (backward) $\eta_{e}$ and is strongly correlated to the one found for the $\bar{d}(\bar{u})$ polarized PDFs in the RHIC kinematic region in contrast to the backward (forward) $\eta_{e}$ region dominated by the behavior of the well-known valence $u(d)$ polarized PDFs [20]. At midrapidity, $W^{+(-)}$production probes a combination of the polarization of the $u$ and $\bar{d}(d$ and $\bar{u})$ quarks, and $A_{L}^{W^{+(-)}}$is expected to be negative (positive). ${ }^{12,27}$ Any measurements beyond this first result will focus on making $\eta_{e}$ dependent asymmetry measurements at midrapidity together with measurements at forward and backward pseudorapidities constraining the polarization of $\bar{d}$ and $\bar{u}$ quarks.

A major step forward has been achieved with the release of preliminary results based on the Run 12 data sample shown in Figure 5 (b) [24]. While the $W^{+}$asymmetry result is consistent with the DSSV prediction, ${ }^{12}$ the $W^{-}$asymmetry result suggests a larger $\bar{u}$ quark polarization. This is indeed the finding from a full global analysis incorporating the Run 12 asymmetry results.

Figure 8 shows the impact on the polarized $u$ and $d$ antiquark distribution functions. ${ }^{17}$ Future RHIC $W$ asymmetry measurements shown in Figure 8 (a) will have a substantial impact in comparison to a global fit result which does not include any $W$ asymmetry results from RHIC by at least a factor 2 compared to the original DSSV fit uncertainties ${ }^{12,27}$ (Figure 8 (b), (c)). The impact of the Run 12 preliminary results suggest a large difference of $x(\Delta \bar{u}-\Delta \bar{d})$ of about 0.07 . This result is intriguing pointing to a broken QCD sea, similar to the unpolarized case. Extending 
(a)

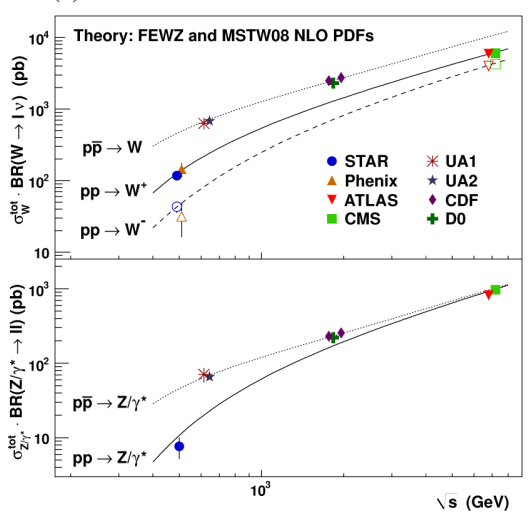

(b)

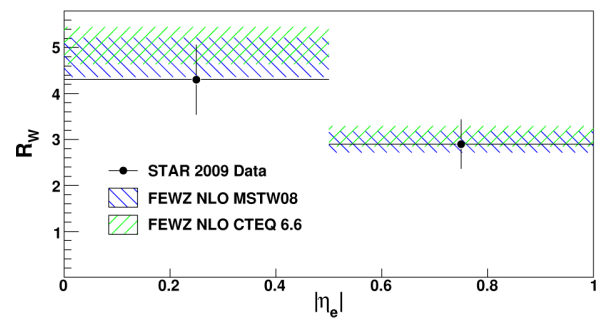

Fig. 7. $W / Z$ cross-section measurements as a function of $\sqrt{s}$ (a) and the $W^{+} / W^{-}$cross-section ratio measurement as a function of $|\eta|$ (b).
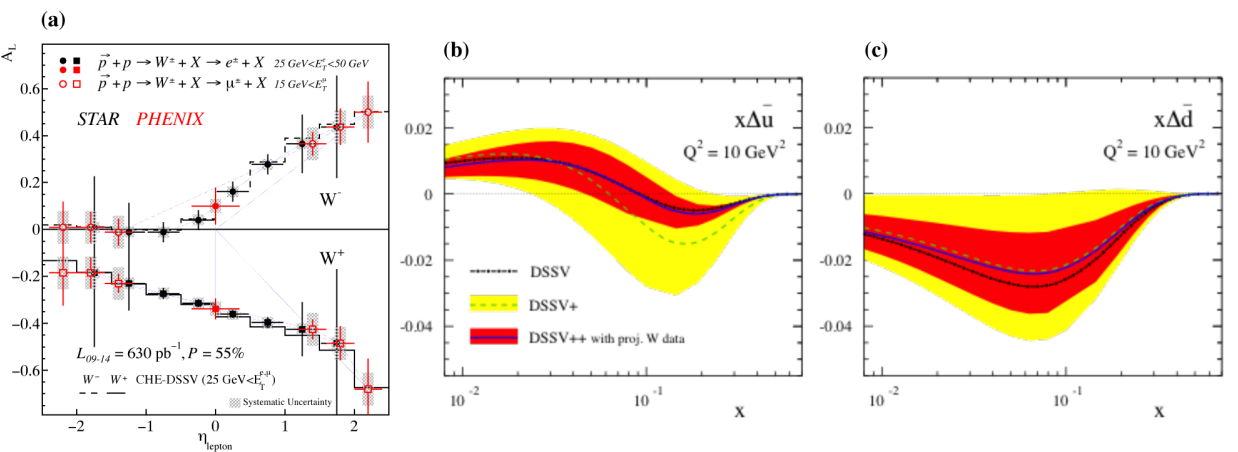

Fig. 8. Impact of RHIC W measurements of $A_{L}$ (a) on antiquark polarizations, $\Delta \bar{u}(b)$ and $\Delta \bar{d}$ (c). The red shaded band is on average at least a factor two smaller compared to the original DSSV uncertainty band. ${ }^{12,27}$

these measurements to backward and forward pseudo-rapidity regions has been a long-standing goal of the RHIC Spin program and is now even more important with the recent finding in Run 12.

\section{Summary}

In summary, new results of jet production at RHIC in polaried proton-proton collision suggest a gluon spin contribution of similar magnitude than the quark spin contribution itself. It is the hope that these findings can be confronted with Lattice QCD calculations. The ground work has been laid by X. Ji et al. in recent papers $[28,29,30]$. Results of $W$ boson production suggest a large asymmetry in the QCD sea, i.e. the difference of the polarization of anti-u and anti-d quarks. Such a scenario has been discussed in the past in the context of a valence scenario by the GRSV group ${ }^{11}$ and in particular by J. Soffer. ${ }^{31,32}$ Recent lattice QCD calculations 
presented by H. Lin in Ref. [33] point to the same finding. The experimental goal of the RHIC Spin program is an extension of the kinematic region at low- $x$ along with an increase of precision for both di-jet production and $W$ boson production in particular in the backward / forward pseudo-rapidity region

\section{References}

1. STAR Collaboration, B. Abelev et al., Phys. Rev. Lett. 97, 252001 (2006).

2. STAR Collaboration, B. Abelev et al., Phys. Rev. Lett. 100, 232003 (2008).

3. STAR Collaboration, L. Adamczyk et al., Phys. Rev. D86, 032006 (2012).

4. STAR Collaboration, B. Abelev et al., Phys. Rev. D80, 111108 (2009).

5. STAR Collaboration, P. Djawotho, arXiv/1106.5769 (2011).

6. STAR Collaboration, M. Walker, arXiv/1107.0917 (2011).

7. G. Bunce, N. Saito, J. Soffer, and W. Vogelsang, Ann. Rev. Nucl. Part. Sci. 50, 525 (2000).

8. B. Jager, M. Stratmann, and W. Vogelsang, Phys. Rev. D70, 034010 (2004).

9. STAR Collaboration, B. Surrow, AIP Conf. Proc. 675, 318 (2003).

10. STAR Collaboration, B. Surrow, PoS - DIS 2010 Conference (Florence, Italy) 1, 249 (2010).

11. M. Gluck, E. Reya, M. Stratmann, and W. Vogelsang, Phys. Rev. D63, 094005 (2001).

12. D. de Florian, R. Sassot, M. Stratmann, and W. Vogelsang, Phys. Rev. Lett. 101, 072001 (2008).

13. D. de Florian, R. Sassot, M. Stratmann, and W. Vogelsang, Prog. Part. Nucl. Phys. 67, 251 (2012).

14. M. Stratmann, Word Scientific - DIS 2006 Conference (Tsukuba, Japan) 1, 715 (2006).

15. D. de Florian, S. Frixione, A. Signer, and W. Vogelsang, Nucl. Phys. B539, 455 (1999).

16. STAR collaboration, T. Sakuma and M. Walker, J. Phys. Conf. Ser. 295, 012068 (2011).

17. E. Aschenauer et al., arXiv/1304.0079 (2013).

18. C. Bourrely and J. Soffer, Phys. Lett. B314, 132 (1993).

19. P. M. Nadolsky and C. Yuan, Nucl. Phys. B666, 31 (2003).

20. D. de Florian and W. Vogelsang, Phys. Rev. D81, 094020 (2010).

21. A. V. Kotwal and J. Stark, Ann. Rev. Nucl. Part. Sci. 58, 147 (2008).

22. STAR Collaboration, M. Aggarwal et al., Phys. Rev. Lett. 106, 062002 (2011).

23. STAR Collaboration, L. Adamczyk et al., Phys. Rev. D85, 092010 (2012).

24. STAR Collaboration, J. R. Stevens, arXiv/1302.6639 (2013).

25. B. Surrow, Nucl. Instrum. Meth. A617, 196 (2010).

26. D. de Florian, G. Navarro, and R. Sassot, Phys. Rev. D71, 094018 (2005).

27. D. de Florian, R. Sassot, M. Stratmann, and W. Vogelsang, Phys. Rev. D80, 034030 (2009).

28. X. Ji, J.-H. Zhang, and Y. Zhao, Phys. Rev. Lett. 111, 112002 (2013).

29. X. Ji, (2013), arXiv/1305.1539 (2013).

30. X. Ji, These Proceedings (2013).

31. C. Bourrely, J. Soffer, and F. Buccella, Eur.Phys.J. C23, 487 (2002).

32. C. Bourrely, F. Buccella, and J. Soffer, arXiv/1308.3567 (2013).

33. H.-W. Lin, These Proceedings (2013). 